\title{
Diversidad de la ictiofauna de la laguna Hule, Costa Rica
}

\author{
José Pablo Barquero-González', Ronald Mora-Vargas², Jorge Valerio-Vargas ${ }^{3} \&$ Juan Ulloa-Rojas ${ }^{3}$ \\ 1. Laboratorio de Sistemática, Genética y Evolución (LabSGE), Escuela de Ciencias Biológicas, Universidad Nacional, Heredia, Costa Rica; \\ jopbgon@gmail.com \\ 2. Programa de Manejo y Conservación de Vida Silvestre Subregion Heredia del Área de Conservación Central, Sistema Nacional de Áreas Prote- \\ gidas (SINAC), Calle Padre Royo, Heredia, Costa Rica: ronald.mora@sinac.go.cr \\ 3. Laboratorio de Acuicultura Continental, Escuela de Ciencias Biológicas, Universidad Nacional, Heredia, Costa Rica; jvaleriovargas@gmail.com, \\ juan.ulloa.rojas@una.cr
}

Recibido 05-IX-2017 • Corregido 10-XI-2017 • Aceptado 24-XI-2017

\begin{abstract}
Ichthyofaunal diversity in Laguna Hule, Costa Rica. Laguna Hule is a volcanic origin crater which has been flooded over time, resulting in a deep mass of water with no previous studies about its fish community. For five months we sampled it and a draining stream (Pata de Gallo) with nets, and hook and line. The captured specimens were identified in situ to the possible lowest taxonomic level. The lagoon mostly had species normally found near aquatic vegetation: Amatitlania siquia, Amphilophus alfari, Astyanax aeneus, Cynodonichthys sp., Parachromis dovii, Phallichthys amates and Poecilia gillii (dominant: $A$. aeneus, $P$. gillii and $P$. amates). The stream was dominated by Brachyrhaphis olomina but also had Rhamdia laticauda. The low diversity indices $(1,48$ in the lagoon and 0,26 in the stream) are characteristic of lacustrine ecosystems. Females were, as expected, larger and more abundant in $P$. amates and $B$. olomina; whereas in $P$. gilli, some males were larger. The area is affected by pollution, introduction of invasive species, uncontrolled fishing and use of destructive fishing gear. We propose demarcation of site protection status and organizing a community group to protect this fish community.
\end{abstract}

Key words: Hule lagoon, ichthyology, fish diversity, fresh water fish

En Costa Rica existe una gran cantidad de lagos asociados al vulcanismo (Gocke, 1997; Alvarado \& Salani, 2004). La laguna Hule se encuentra localizada en una depresión volcánica ocupada por tres lagos, de los cuales Hule es el más grande ocupando $54,7 \mathrm{Ha}$ y una profundidad de $26,5 \mathrm{~m}$. La zona de vida presente en la región es el bosque muy húmedo premontano, con una precipitación media anual del área de 4500-5500mm (Holdridge, 1987).

En las comunidades de organismos acuáticos, uno de los grupos de mayor importancia son los peces, cuyos
RESUMEN: La laguna Hule es un cráter de origen volcánico que se ha inundado con el tiempo, dando como resultado una gran masa de agua, sin estudios previos sobre su comunidad de peces. Muestreamos durante cinco meses la laguna y una quebrada (Pata de Gallo) con redes, gancho y línea. Los especímenes capturados se identificaron in situ al nivel taxonómico posible más bajo. La laguna tenía especies que normalmente se encuentran cerca de la vegetación acuática: Amatitlania siquia, Amphilophus alfari, Astyanax aeneus, Cynodonichthys sp., Parachromis dovii, Phallichthys amates y Poecilia gillii (dominante: $A$. aeneus, P. gillii y P. amates). La quebrada estaba dominada por Brachyrhaphis olomina pero también tenía Rhamdia laticauda. Los índices de baja diversidad (1,48 en la laguna y 0,26 en la quebrada) son característicos de los ecosistemas lacustres. Las hembras fueron, como se esperaba, más grandes y abundantes en $P$. amates y $B$. olomina; mientras que en $P$. gilli, algunos machos eran más grandes. El área se ve afectada por la contaminación, la introducción de especies invasoras, la pesca incontrolada y el uso de equipo destructivo de pesca. Proponemos la demarcación del estado de protección del sitio y la organización de un grupo comunitario para proteger a esta comunidad de peces.

Palabras clave: Laguna Hule, ictiología, diversidad de peces, peces de agua dulce mecanismos de supervivencia comprenden adaptaciones morfológicas, fisiológicas, reproductivas y alimentarias, que repercuten directa o indirectamente en los hábitats que frecuentan (Jackson, Peres-Neto \& Olden, 2001). La ictiofauna continental neotropical se caracteriza por poseer el $24 \%$ de la totalidad de los peces a nivel mundial (Albert \& Reis, 2011). Esta riqueza se ve representada en los diferentes ambientes (loticos, lenticos o estuarinos) de la región y en Costa Rica convergen tres de las cuatro provincias ícticas, factor que explica su considerable diversidad (Bussing, 1998; Angulo et al., 2013). 
Los peces juegan un papel importante en las dinámicas tróficas de los cuerpos de agua, donde a través de la depredación del zooplancton promueven efectos de cascada trófica (Carpenter \& Kitchell, 1996; Frank et al., 2005; Kratina et al., 2012). Además, conectan diferentes zonas mediante la translocación de nutrientes entre hábitats (Vander Zanden \& Vadeboncoeur, 2002). Permiten el desarrollo del perifiton y el fitoplancton (Jones \& Sayer, 2003, p. 2156) al proveerlos con nutrientes excretados (Scheffer et al., 1993; Vanni, 2002; Tessier \& Woodruff, 2002; Ellis et al., 2011).

Una de las formas de describir una comunidad ecológica es determinando su composición de especies y la abundancia relativa de cada una. Los índices de diversidad ayudan a resumir las asociaciones de organismos (Wilhm, 1968; Mason et al., 2005). Los cambios en la composición de especies son indicadores de presiones en el ecosistema y de los impactos ambientales a través del tiempo (Livingston, 1976; Moore, 1978; Smith, 1991; Weibull, Östman \& Granqvist, 2003; Gorelick, 2006). Se registran pocos trabajos relacionados con peces en este lago (Tabash, Farid \& Guadamuz 2000), de modo que este es el primer estudio descriptivo de la comunidad íctica en la Laguna del Hule.

\section{MATERIALES Y MÉTODOS}

La laguna Hule se ubica en una depresión volcánica a $800 \mathrm{msnm}$, en la Vertiente Caribe de la Cordillera Volcánica Central, en el cantón de Río Cuarto, Alajuela, Costa Rica; forma parte del Refugio Nacional de Vida Silvestre Bosque Alegre. La investigación se realizó con el permiso de investigación del SINAC No. 03116. El muestreo se hizo dos veces al mes, en el día y la noche, durante la temporada lluviosa de agosto a diciembre del 2015. El muestreo también incluyó el efluente quebrada Pata de Gallo, que representa el cuerpo de agua más cercano a la laguna.

La abundante cantidad de vegetación acuática condicionó el muestreo a los siguientes artes de pesca: líneas de pesca, redes y chinchorro de diferente luz de malla. Se muestreó en la orilla expuesta y entre la vegetación un tramo total de aproximadamente $800 \mathrm{~m}$, mismos que se dividieron en ocho secciones de cien metros cada una, en promedio se muestreaba durante $20 \mathrm{~min}$ en cada sección. Los peces capturados fueron identificados in situ utilizando la clave taxonómica elaborada por Bussing, (1998), se midió su longitud estándar (LS) usando un ictiómetro $( \pm 1 \mathrm{~mm})$ o una regla plástica $( \pm 0,5 \mathrm{~mm})$ cuando su tamaño lo condicionaba y se liberaron en el mismo sitio donde se capturaron. Además, se verificó el sexo de los poecílidos.

El aporte de las especies dominantes se determinó mediante el criterio de Ayala-Pérez et al. (1998), quienes lo establecen como el aporte de más del $80 \%$ de la captura total. Para el procesamiento estadístico y la creación de gráficos se utilizó el software libre R (R Development Core Team, 2008) y Excel. Se evaluaron algunas relaciones ecológicas utilizando índices de diversidad y el paquete estadístico "Vegan" del software R. Los índices elegidos para la abundancia relativa fueron los de Pielou, Fischer, Soresen y especies no detectadas; además se generó una curva de acumulación de especies.

Para determinar si las diferencias entre el tamaño de machos y hembras de las especies de poecílidos eran significativas se aplicó la prueba de Welch Two Sample t-test. En caso que los datos no presentaran homogeneidad de varianza, se aplicó un test de Wilcoxon; misma prueba que se utilizó para buscar preferencias en el uso del hábitat de las especies capturadas. Además, se evaluó visualmente el estado ribereño de la laguna en busca acciones antropogénicas que pudieran amenazar la integridad y biodiversidad del sitio, cuantificando las principales problemáticas presentes para su posterior discusión.

\section{RESULTADOS}

Seis especies se registraron en las orillas de la Laguna Hule y dos en la quebrada Pata de Gallo, para un total de 223 y 117 individuos, respectivamente (Cuadro 1). Las especies más abundantes en la laguna correspondieron a A.aeneus, P.amates y P.gillii, mientras que en la quebrada fue $B$. olomina. La frecuencia relativa con la que se encontraron las especies fue de 0,013 para A. alfari, 0,25 para $A$. aeneus, 0,184 para $P$. dovii, 0,197 para $P$. amates y 0,348 para P. gillii.

Un evento de relevancia es el primer reporte de la presencia del género Cynodonichtys en el sitio, específicamente en charcas temporales cerca de la laguna, siendo la zona más cercana donde se ha reportada el Río Sarapiquí, a una distancia de $4 \mathrm{~km}$ de la laguna. Además, se informa de la presencia de $A$. siquia en la laguna Congo, que a pesar de la cercanía con la laguna Hule, no fue encontrada en la misma durante todos los meses de muestreo. Relacionado con la elección de hábitat (Fig. 1), se determinó que las especies A. alfari, P. amates, P. gillii (t-test $(p<0.05))$ y P. dovii (Wilcoxon $(p>0.05))$ muestran una tendencia marcada a vivir resguardadas entre la vegetación acuática o de la orilla, mientras que A. aeneus posee una preferencia hacia aguas abiertas (t-student $(p<0.05))$. 


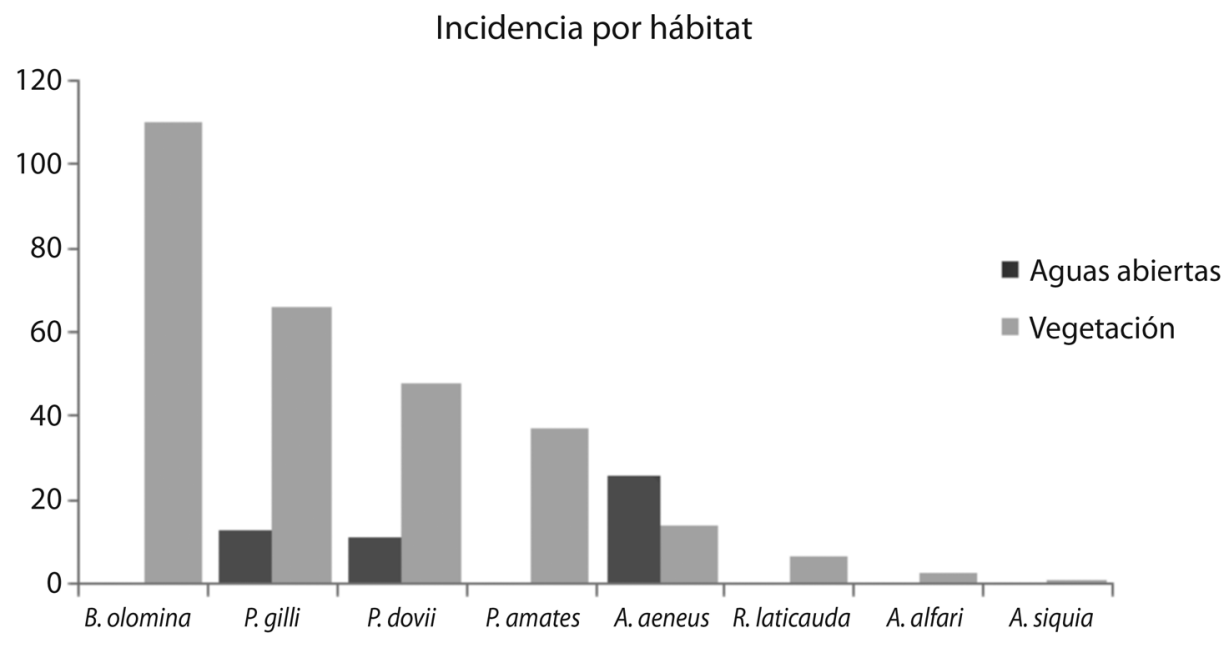

Fig. 1. Incidencia de captura de cada especie por hábitat.

CUADRO 1

Especies, cantidad de ejemplares capturados, y tallas de peces en la Laguna de Hule y en el efluente Pata de Gallo.

\begin{tabular}{lcccc}
\multicolumn{1}{c}{ Especies } & Hule & Pata de Gallo & \multicolumn{2}{c}{ Longitud estándar (cm) } \\
Amatitlania siquia* & 2 & 0 & 6 & 9.5 \\
Amphilophus alfari & 3 & 0 & 2.3 & 20 \\
Astyanax aeneus & 40 & 0 & 1.9 & 14 \\
Parachromis dovii & 59 & 0 & 4 & 2.7 \\
Phallichthys amates & 37 & 0 & 2.5 & 6.1 \\
Poecilia gillii & 79 & 0 & 5.3 & 8.3 \\
Brachyrhaphis olomina & 0 & 110 & 8.9 & 8.6 \\
Rhamdia laticauda $_{\text {Cynodonichthys sp. }}^{* *}$ & 0 & 7 & - & 14.5 \\
Total & 3 & 0 & 117 & - \\
\hline
\end{tabular}

*Capturado en una visita a la Laguna Congo.

** Capturado en charcos cerca de la laguna.

En las tres especies de poecílidos las hembras son más abundantes que los machos, con frecuencias que varían entre 0.7 y 0.83 , siendo la más alta para $B$. olomina, que se encontró en la quebrada, donde la frecuencia de machos (0.17) es la más baja. De todos los individuos muestreados, se encontró que los de esta quebrada mostraban parásitos dermales en forma de manchas negras, en una frecuencia de 0.16. Mientras que en la laguna Hule se observó que la mayor parte de los juveniles de $P$. dovii presentaron gran cantidad de quistes dermales.

No hubo diferencia entre el tamaño promedio de los sexos para P. gillii y P. amates (Welch Two Sample, t-test $(\mathrm{p}>0.05))$, caso contrario B. olomina si presentó diferencias (Welch Two Sample t-test $(p<0.05))$. En general, las hembras de $B$. olomina y $P$. amates presentaron mayores tallas, al contrario que con $P$. gillii (machos de mayor tamaño) (Cuadro 2).

Los índices de diversidad en los dos sitios muestran que esta es baja, siendo la Laguna de Hule la más diversa de las dos; el índice de equidad de Pielou refleja una dominancia bastante repartida en la laguna; mientras que en la quebrada la alta dominancia por parte de una especie es muy notable. Ninguna de las especies encontradas en la quebrada estaba presente en la Laguna de Hule y viceversa, aspecto que se confirma con el índice de disimilitud de Soresen. La curva de acumulación de especies 


\section{CUADRO 2}

Evaluación del promedio de la longitud estándar (LS) de las especies de poecílidos, según su sexo.

\begin{tabular}{lcc}
\multicolumn{1}{c}{ Especie } & LS hembras $(\mathrm{cm})$ & LS machos $(\mathrm{cm})$ \\
B. olomina & $6.528 \mathrm{a}$ & $5.278 \mathrm{~b}$ \\
P. gilli & $4.302 \mathrm{a}$ & $4.823 \mathrm{a}$ \\
P. amates & $4.208 \mathrm{a}$ & $4.109 \mathrm{a}$ \\
\hline
\end{tabular}

Promedios con diferente letra, en la misma fila, presentaron diferencia significativa $(p<0.05)$.

\section{CUADRO 3}

Valores de los índices ecológicos aplicados a las poblaciones de peces capturados en los diferentes sitios muestreados durante un periodo de 5 meses en la laguna Hule.

\begin{tabular}{lcc}
\multicolumn{1}{c}{ Índices } & Hule & Pata de Gallo \\
Shannon & 1,4839 & 0,2265 \\
Pielou & 0,7626 & 0,3268 \\
Fisher & 1,3737 & 0,3427 \\
Soresen & 1 & \\
Acumulación Especies & 6,5636 & 2 \\
\multicolumn{4}{l}{ Jack knife } & Índices de especies no detectadas \\
Bootstrap & 2,92 & \\
\hline
\end{tabular}

señala que la mayor parte de estas fue hallada en la laguna. Según los índices de especies no detectadas, faltaron de tres a cinco de encontrar en la laguna Hule (Cuadro 3). Los problemas de origen antropogénico encontrados se resumen en el Cuadro 4, así como sus riesgos y posibles soluciones.

\section{DISCUSIÓN}

La baja diversidad de especies encontrada en los sitios de muestreo manifiesta que los hábitats estudiados no poseen la suficiente heterogeneidad, por lo que no proveen una mayor disponibilidad de micro-hábitats que permitan una mayor riqueza y colonización de especies (Walter \& Haynes, 2006; Matthews, 2012). La heterogeneidad estructural del hábitat moldea la estructura de la comunidad de peces tropicales, influyendo en la distribución de especies y en los patrones de segregación a diversas escalas temporales (Ault \& Johnson, 1998; Palmer, Menninger \& Bernhardt, 2010). Las especies encontradas son propias de aguas mansas y con poca corriente, presentan una preferencia por ambientes profundos y anchos, con una menor velocidad de agua (Bart, 1989; Matthews, 2012). Los hábitats profundos proporcionan mayor estabilidad que los que presentan rápidos, debido a que atenúan cambios en la velocidad de corriente, profundidad y diferencias en el sustrato; lo cual pudo influenciar las diferencias entre la laguna y la quebrada (Schlosser, 1982; Beeton, 2002).

A diferencia de quebradas y ríos, donde las especies base en la cadena trófica, como A. aeneus, tienden a refugiarse en rápidos para evitar ser depredadas; en las lagunas, estas se distribuyen en aguas abiertas. Lo anterior disminuye la probabilidad de encontrarse con depredadores acuáticos que acechan en la maleza, como el P. dovii (Bussing, 1998; Mehner et al., 2005). Los peces de la familia Characidae son capaces de habitar regiones con mayor elevación y flujo de caudal, permitiéndoles un mayor ámbito de distribución en los cuerpos de agua dulce (Bussing \& López-Sánchez, 1977; Azevedo, Melo \&

CUADRO 4

Lista de problemáticas encontradas en la laguna de Hule durante los muestreos, riesgos que conllevan y posibles soluciones.

\begin{tabular}{lll}
\multicolumn{1}{c}{ Problemáticas } & \multicolumn{1}{c}{ Riesgos } & \multicolumn{1}{c}{ Posibles soluciones } \\
$\begin{array}{l}\text { Contaminación por parte } \\
\text { de los visitantes (equipo de } \\
\text { pesca abandonado, latas, } \\
\text { basura en general) }\end{array}$ & $\begin{array}{l}\text { Los animales corren riesgos de ingestión, quedar } \\
\text { atrapados o ser heridos, pérdida de la belleza } \\
\text { escénica. }\end{array}$ & $\begin{array}{l}\text { Acondicionar basureros en el sitio, rótulos recordando } \\
\text { al visitante sus responsabilidades, crear un grupo de } \\
\text { limpieza que visite regularmente el sitio }\end{array}$ \\
$\begin{array}{l}\text { Desconocimiento del estatus } \\
\text { de protección de la zona }\end{array}$ & $\begin{array}{l}\text { Se realizan actividades no permitidas en lugares } \\
\text { protegidos (pesca, caza, contaminación) }\end{array}$ & $\begin{array}{l}\text { Creación y colocación de rótulos aclarando el estatus de } \\
\text { protección de la zona. }\end{array}$ \\
$\begin{array}{l}\text { Introducción de especies } \\
\text { invasivas }\end{array}$ & $\begin{array}{l}\text { Desbalance en la cadena trófica que puede } \\
\text { poner en riesgo la supervivencia de varias } \\
\text { especies y comprometer la estabilidad del } \\
\text { ecosistema }\end{array}$ & $\begin{array}{l}\text { Impartir talleres en la zona advirtiendo los riesgos que } \\
\text { implica introducir especies en el lago }\end{array}$ \\
$\begin{array}{l}\text { Pesca no controlada y } \\
\text { utilización de artes de }\end{array}$ & $\begin{array}{l}\text { Disminución de las poblaciones de las especies } \\
\text { pescadadas destructivos }\end{array}$ & $\begin{array}{l}\text { Colocar rótulos donde explícitamente se indique la } \\
\text { ilegalidad de la actividad y las medidas legales (multas } \\
\text { u otras); crear un grupo comunal que vigile la zona varias } \\
\text { veces a la semana }\end{array}$ \\
\hline
\end{tabular}


Young, 2011; Kasumyan \& Marusov, 2015). A. aeneus además es muy importante en la red trófica, dado su papel en el ecosistema como uno de los principales recicladores de nutrientes (nitrógeno y fósforo), en hábitats donde hay poco aporte de los mismos (Small et al., 2011).

Los poecílidos presentes siguen la tendencia de esta familia de peces, predominando las hembras en número y tamaño (Chapman et al., 1991; Jennions \& Kelly, 2002). El hábitat de preferencia es consistente con el reportado (Bussing, 1998), P. amates solo se encontró en la vegetación acuática; $P$. gillii es más adaptable, pudiendo vivir en aguas rápidas con caudales moderados (Burcham, 1988; Lorion, Kennedy \& Braatne, 2011). A pesar de que se reporta un mayor tamaño para hembras de $P$. gillii (Bussing, 1998), estas observaciones muestran que algunos machos sobrepasan el tamaño máximo de las hembras. Respecto a las demás especies, sus tamaños máximos y mínimos están dentro de los rangos previamente reportados (Angulo et al., 2013). Para P. dovii no se contaron con muchos datos, debido a la imposibilidad de muestrear lejos de la orilla (ausencia de botes y sus respectivos permisos para utilizarlos).

Un hallazgo importante para el Refugio Bosque Alegre es el primer reporte de la presencia de Cynodonichthys, cuyo hábitat tipo consiste en charcos lodosos, pantanos, acequias y aguas de poca a moderada velocidad de corriente; coincidiendo con el tipo de hábitat donde fue encontrado en este trabajo (charcos lodosos de origen volcánico de poca profundidad cercanos a la laguna) (Bussing 1998).

Se encontró que la población de B. olomina poseía longitudes estándar que superan las reportadas por Bussing (1998) $(60 \mathrm{~mm}$ frente a $75 \mathrm{~mm})$; diferencia que puede atribuirse a la escasez de depredadores. Aunque las poblaciones de Brachyrhaphis ocupen distintos hábitats, las diferencias ecológicas más pronunciadas entre ellas son causadas por la presencia o ausencia de peces depredadores. A excepción de R. laticauda, no se encontró ningún pez en la quebrada que pudiese alimentarse de esta especie, por lo que la mortalidad debe estar asociada a otros factores ecológicos o depredadores terrestres (Johnson, 2002). Se observó la presencia de parásitos dérmicos en un $25 \%$ de la población de B. olomina, que pudiesen ser los encargados de controlar en parte el tamaño de su población.

La Laguna de Hule presenta diversas problemáticas ambientales. La falta de vigilancia y estricta demarcación de la zona son las causas principales de la pesca y contaminación. Este es un tema complicado ya que la naturaleza mixta público-privado del refugio no permite el suficiente control sobre los asuntos relacionados a su manejo. En los múltiples muestreos realizados se encontró una gran cantidad de basura dispersa dentro de la laguna y en sus alrededores, patrón de comportamiento común en varios lagos (Corona-Lisboa, 2013). Se propone la colocación de rótulos, que delimiten el estatus de protección de la zona. Dichas señalizaciones deberían distribuirse a lo largo del camino, en distintas ubicaciones, para evitar que sean robados o removidos. Además, se propone la creación de un grupo comunal que vigile la laguna varias veces a la semana.

Se encontraron personas realizando actividades de pesca durante todos los muestreos. La pesca del $P$. dovii se lleva a cabo sin regulaciones. Tabash, Farid \& Guadamuz (2000) mostraron que un incremento en la pesca deportiva de esta especie en la laguna podría afectar la capacidad de recuperación de la población. Esto puede propiciar fluctuaciones peligrosas en las poblaciones, ocasionadas por el descenso del número de individuos maduros y la consecuente baja en su capacidad reproductiva (Murawski, 2000; Jackson et al., 2001; Allan et al., 2005). Esta pesca casi siempre es acompañada de la extracción de sardinas (A. aeneus) para utilizarlas como carnada, con el consecuente efecto sobre esta especie.

Los habitantes constataron la liberación de especies invasoras en este cuerpo de agua. Puntualmente se liberaron especies como el machín (Agonostomus montícola) y sábalo (Brycon guatemalensis), lo cual no se pudo corroborar en los muestreos. Esta situación podría llevar a un desbalance en la cadena trófica, poniendo en riesgo la supervivencia de varias especies (Pimentel, Zuñiga \& Morrison, 2005). Se propone impartir talleres en la zona, advirtiendo los riesgos que implica introducir especies en la laguna (Benayas, Gutiérrez, \& Hernández, 2003).

\section{REFERENCIAS}

Albert, J. S., \& Reis, R. (Eds.). (2011). Historical biogeography of Neotropical freshwater fishes. California, United States of America: University of California Press.

Allan, J. D., Abell, R., Hogan, Z. E. B., Revenga, C., Taylor, B. W., Welcomme, R. L., \& Winemiller, K. (2005). Overfishing of inland waters. BioScience, 55(12), 1041-1051. doi: 10.1641/0006-3568(2005)055[1041:OOIW]2.0.CO;2

Alvarado, G. E., \& Salani, F. M. (2004). Tefroestratigrafía (40 000$2000 \mathrm{aP}$ ) en el sector caribe de los volcanes Barva, Congo y Hule, Cordillera Central, Costa Rica. Revista Geológica de América Central, 30, 59-72. doi: 10.15517/ rgac.v0i30.7254

Angulo, A., Garita-Alvarado, C. A., Bussing, W. A., \& López, M. I. (2013). Annotated checklist of the freshwater fishes of continental and insular Costa Rica: additions and 
nomenclatural revisions. Check List, 9(5), 987-1019. doi: 10.15560/9.5.987

Ault, T. R., \& Johnson, C. R. (1998). Spatial variation in fish species richness on coral reefs: habitat fragmentation and stochastic structuring processes. Oikos, 82 (2), 354-364. doi: $10.2307 / 3546976$

Ayala-Pérez, L. A., Avilés-Alatriste, O. A., \& Rojas-Galaviz, J. L. (1998). Estructura de la comunidad de peces en el sistema Candelaria-Panlau, Campeche, México. Revista de Biología Tropical, 46(3), 763-774. Recuperado de: http:// revistas.ucr.ac.cr/index.php/rbt/article/view/20452

Azevedo, P. G., Melo, R., \& Young, R. J. (2011). Feeding and social behavior of the piabanha, Brycon devillei (Castelnau, 1855) (Characidae: Bryconinae) in the wild, with a note on following behavior. Neotropical Ichthyology, 9(4), 807-814. doi: 10.1590/S1679-62252011005000046

Bart, H. L. (1989). Fish habitat association in an Ozark stream. Environmental Biology of Fishes, 24(3), 173-186. doi: 10.1007/BF00001222

Beeton, A. M. (2002). Large freshwater lakes: present state, trends, and future. Environmental Conservation, 29(01), 21-38. doi: 10.1017/S0376892902000036

Benayas, J., Gutiérrez, J., \& Hernández, N. (2003). La investigación en educación ambiental en España. España: Ministerio de Medio Ambiente, Organismo Autónomo Parques Nacionales.

Burcham, J. (1988). Fish communities and environmental characteristics of two lowland streams in Costa Rica. Revista de Biología Tropical, 36(2), 273-285. Recuperado de: https://revistas.ucr.ac.cr/index.php/rbt/article/view $/ 23711$

Bussing, W. A. (1998). Freshwater fishes of Costa Rica (Vol. 46). San José , Costa Rica: Editorial Universidad de Costa Rica.

Bussing, W. A., \& López-Sánchez, M. I. (1977). Distribución y aspectos ecológicos de los peces de las cuencas hidrográficas de Arenal, Bebedero y Tempisque, Costa Rica. Revista de Biología Tropical, 25(1), 13-37. doi: 10.15517/rbt.v25i1.25842

Carpenter, S. R., \& Kitchell, J. F. (1996). The trophic cascade in lakes. United Kingdom, England: Cambridge University.

Chapman, L. J., Kramer, D. L., \& Chapman, C. A. (1991). Population dynamics of the fish Poecilia gillii (Poeciliidae) in pools of an intermittent tropical stream. The Journal of Animal Ecology, 60(2), 441-453. doi: 10.2307/5289

Corona-Lisboa, J. L. (2013). Contaminación antropogénica en el lago de Maracaibo, Venezuela. Biocenosis, 27 (1-2). Recuperado de: http://investiga.uned.ac.cr/revistas/index.php/biocenosis/article/view/614/509

Ellis, B. K., Stanford, J. A., Goodman, D., Stafford, C. P., Gustafson, D. L., Beauchamp, D. A., ... \& Hansen, B. S. (2011). Longterm effects of a trophic cascade in a large lake ecosystem. Proceedings of the National Academy of Sciences, 108(3), 1070-1075. doi: 10.1073/pnas.1013006108
Frank, K. T., Petrie, B., Choi, J. S., \& Leggett, W. C. (2005). Trophic cascades in a formerly cod-dominated ecosystem. Science, 308(5728), 1621-1623. doi: 10.1126/ science.1113075

Gocke, K. (1997). Basic morphometric and limnological properties of Laguna Hule, a caldera lake in Costa Rica. Revista de Biología Tropical, 44(45), 537-548. doi: 10.15517/rbt. v44i3.30665

Gorelick, R. (2006). Combining richness and abundance into a single diversity index using matrix analogues of Shannon's and Simpson's indices. Ecography, 29(4), 525530. doi:10.1111/j.0906-7590.2006.04601.x

Holdridge, L. R. (1987). Ecología basada en zonas de vida (No. 83). San José, Costa Rica: Agroamérica.

Jackson, J. B., Kirby, M. X., Berger, W. H., Bjorndal, K. A., Botsford, L. W., Bourque, B., \& Hughes, T. P. (2001). Historical overfishing and the recent collapse of coastal ecosystems. Science, 293(5530), 629-637. doi: 10.1126/ science.1059199

Jackson, D. A., Peres-Neto, P. R., \& Olden, J. D. (2001). What controls who is where in freshwater fish communities the roles of biotic, abiotic, and spatial factors. Canadian Journal of Fisheries and Aquatic Sciences, 58(1), 157-170. doi: 10.1139/f00-239

Jennions, M. D., \& Kelly, C. D. (2002). Geographical variation in male genitalia in Brachyrhaphis episcopi (Poeciliidae): is it sexually or naturally selected? Oikos, 97(1), 79-86. doi: 10.1034/j.1600-0706.2002.970108.x

Johnson, J. B. (2002). Divergent life histories among populations of the fish Brachyrhaphis rhabdophora: detecting putative agents of selection by candidate model analysis. Oikos, 96(1), 82-91. doi: 10.1034/j.1600-0706.2002.960109.x

Jones, J. I., \& Sayer, C. D. (2003). Does the fish-invertebrateperiphyton cascade precipitate plant loss in shallow lakes? Ecology, 84(8), 2155-2167. doi: 10.1890/02-0422

Kasumyan, A. O., \& Marusov, E. A. (2015). Chemoorientation in the feeding behavior of the blind Mexican cavefish Astyanax fasciatus (Characidae, Teleostei). Russian Journal of Ecology, 46(6), 559-563. doi:https://doi. org/10.1134/S1067413615060053

Kratina, P., Greig, H. S., Thompson, P. L., Carvalho-Pereira, T. S., \& Shurin, J. B. (2012). Warming modifies trophic cascades and eutrophication in experimental freshwater communities. Ecology, 93(6), 1421-1430. doi: 10.1890/11-1595.1

Livingston, R. J. (1976). Diurnal and seasonal fluctuations of organisms in a north Florida estuary. Estuarine and Coastal Marine Science, 4(4), 373-400. doi: 10.1016/0302-3524(76)90014-1

Lorion, C. M., Kennedy, B. P., \& Braatne, J. H. (2011). Altitudinal gradients in stream fish diversity and the prevalence of diadromy in the Sixaola River basin, Costa Rica. Environmental Biology of Fishes, 91(4), 487-499. doi: 10.1007/s10641-011-9810-6 
Mason, N. W., Mouillot, D., Lee, W. G., \& Wilson, J. B. (2005). Functional richness, functional evenness and functional divergence: the primary components of functional diversity. Oikos, 111(1), 112-118. doi: 10.1111/j.0030-1299.2005.13886.x

Matthews, W. J. (2012). Patterns in freshwater fish ecology. New York: Springer Science \& Business Media.

Mehner, T., Diekmann, M., Brämick, U., \& Lemcke, R. (2005). Composition of fish communities in German lakes as related to lake morphology, trophic state, shore structure and human-use intensity. Freshwater Biology, 50(1), 7085. doi: 10.1111/j.1365-2427.2004.01294.x

Moore, D. M. (1978). Seasonal changes in distribution of intertidal macrofauna in the lower Mersey Estuary, UK. Estuarine and Coastal Marine Science, 7(2), 117-125. doi: 10.1016/0302-3524(78)90069-5

Murawski, S. A. (2000). Definitions of overfishing from an ecosystem perspective. ICES Journal of Marine Science: Journal du Conseil, 57(3), 649-658. doi: 10.1006/jmsc.2000.0738

Palmer, M. A., Menninger, H. L., \& Bernhardt, E. (2010). River restoration, habitat heterogeneity and biodiversity: a failure of theory or practice? Freshwater Biology, 55(s1), 205-222. doi: 10.1111/j.1365-2427.2009.02372.x

Pimentel, D., Zuniga, R., \& Morrison, D. (2005). Update on the environmental and economic costs associated with alien-invasive species in the United States. Ecological Economics, 52(3), 273-288. doi: https://doi.org/10.1016/j. ecolecon.2004.10.002

R Development Core Team (2008). R: A language and environment for statistical computing. $\mathrm{R}$ Foundation for Statistical Computing, Vienna, Austria. Recuperado de http://www.R-project.org.

Scheffer, M., Hosper, S. H., Meijer, M. L., Moss, B., \& Jeppesen, E. (1993). Alternative equilibria in shallow lakes. Trends in Ecology \& Evolution, 8(8), 275-279. doi: 10.1016/0169-5347(93)90254-M

Schlosser, I. J. (1982). Fish community structure and function along two habitat gradients in a headwater stream. Ecological Monographs, 52(4), 395-414. doi: $10.2307 / 2937352$
Small, G. E., Pringle, C. M., Pyron, M., \& Duff, J. H. (2011). Role of the fish Astyanax aeneus (Characidae) as a keystone nutrient recycler in low-nutrient Neotropical streams. Ecology, 92(2), 386-397. doi: 10.1890/10-0081.1

Smith, M. L. (1991). Environmental impact assessment: the roles of predicting and monitoring the extent of impacts. Marine and Freshwater Research, 42(5), 603-614. doi: 10.1071/MF9910603

Tabash, B., Farid, A., \& Guadamuz, S. (2000). A management plan for the sport fishery of Parachromis dovii (Pisces: Cichlidae) (Gunther 1864) in Hule lake, Costa Rica. Revista de Biología Tropical, 48(2-3), 473-485. Recuperado de: https://revistas.ucr.ac.cr/index.php/rbt/ article/view/18816

Tessier, A. J., \&Woodruff, P. (2002). Cryptic trophic cascade along a gradient of lake size. Ecology, 83(5), 1263-1270. doi: 10.1890/0012-9658(2002)083[1263:CTCAAG]2.0.CO;2

Vanni, M. J. (2002). Nutrient cycling by animals in freshwater ecosystems. Annual Review of Ecology and Systematics, 33(1), 341-370. doi: 10.1146/annurev. ecolsys.33.010802.150519

Vander Zanden, M. J., \& Vadeboncoeur, Y. (2002). Fishes as integrators of benthic and pelagic food webs in lakes. Ecology, 83(8), 2152-2161. doi: 10.1890/0012-9658(2002)083[2152:FAIOBA]2.0.CO;2

Walter, R. P., \& Haynes, J. M. (2006). Fish and coral community structure are related on shallow water patch reefs near San Salvador, Bahamas. Bulletin of Marine Science, 79(2), 365-374. Recuperado de: http:// www.ingentaconnect.com/content/umrsmas/ bullmar/2006/00000079/00000002/art00009

Weibull, A. C., Östman, Ö., \& Granqvist, Å. (2003). Species richness in agroecosystems: the effect of landscape, habitat and farm management. Biodiversity and Conservation, 12(7), 1335-1355. doi: 10.1023/A:1023617117780

Wilhm, J. L. (1968). Use of biomass units in Shannon's formula. Ecology, 49(1), 153-156. doi: 10.2307/1933573 